\title{
«No estuvimos a la altura de la situación»: límites de la participación ciudadana en la mesa de diálogo de Espinar (2012-2013)
}

\author{
Lorena De la Puente Burlando*
}

* Licenciada en Sociología por la Pontificia Universidad Católica del Perú y magíster en Estudios Latinoamericanos por la Universidad de Oxford. Investigadora asociada de la oficina regional del Natural Resource Governance Institute (NRGI). Correo electrónico: lorena.delapuenteburlando@gmail.com

Fecha de recepción: 01/03/18. Fecha de aceptación: 31/01/19 


\title{
«No estuvimos a la altura de la situación»: límites de la participación ciuda- dana en la mesa de diálogo de Espinar (2012-2013)
}

\section{RESUMEN}

La mesa de diálogo en Espinar es reconocida como un caso emblemático de procesos de diálogo. Sin embargo, hubo tres barreras que comprometieron la calidad de la representación de la sociedad civil: (i) la sobrecarga para los dirigentes urbanos; (ii) la subrepresentación de los intereses campesinos, y (iii) el uso de lenguaje técnico para regular la toma de decisión. El estudio de este caso pretende explorar los límites y contradicciones de los procesos participativos tras los conflictos socioambientales en el Perú. El objetivo es contribuir con la revisión de sus metodologías y cuestionar los horizontes de sentido desde los cuales parten los distintos actores involucrados.

Palabras clave: minería, gobernanza, participación ciudadana.

\section{"We were not up to the situation": limits of citizen participation in the Espinar's dialogue table (2012-2013)}

\begin{abstract}
The dialogue table in Espinar is recognized as an emblematic case of dialogue processes. However, three barriers compromised the quality of representation of civil society: the overloading of urban leaders, the underrepresentation of peasant interests and the use of technical languages to regulate decision-making. The study of this case aims to explore the limits and contradictions of participatory processes after socio-environmental conflicts in Peru. The objective is to contribute to the revision of their methodologies and to question the ideas from which different stakeholders built their participation.
\end{abstract}

Keywords: mining, governance, civil society participation. 


\section{INTRODUCCIÓN}

A partir del año 2003, el rol de las industrias extractivas en daños al medio ambiente, modos de vida y economías locales se ha encontrado en el centro de la discusión desde que la actividad minera y energética ha incrementado su intensidad operativa en el Perú y en el resto de América Latina (Bebbington, 2013). Como resultado, cientos de conflictos socioambientales se han desarrollado (Núnez et al., 2015) y nuestro país no ha sido la excepción. Desde 2006 hasta la fecha se han registrado cientos de heridos, detenidos y cerca de trescientas pérdidas humanas (El Comercio, 2018), y se estima que en la actualidad existen 181 conflictos (Defensoría del Pueblo, 2019) ${ }^{1}$.

Los conflictos socioambientales en el Perú han tenido como una agenda importante la superación de deficiencias en la prevención estatal de impactos socioambientales generados por empresas principalmente mineras (Oficina Nacional de Diálogo y Sostenibilidad, 2013; Defensoría del Pueblo, 2017).

En la provincia cusqueña de Espinar, reclamos contra el desempeño de una compañía a cargo de uno de los principales proyectos mineros del país desencadenaron un fuerte conflicto en 2012 (El Comercio, 2012), el cual produjo muertes y afectaciones físicas y judiciales contra los protestantes (La República, 2012). Entre demandas por una mayor y mejor distribución de la renta minera, la sociedad civil, en coordinación con el municipio provincial, denunció deficiencias en la fiscalización ambiental. Tras varios reclamos no atendidos, se optó por la movilización, la cual se tornó violenta (Flores, 2016). Según los dirigentes espinarenses, las deficiencias en el manejo ambiental de la compañía habían afectado la salud de los habitantes de las áreas de influencia y sus recursos naturales (Amnistía Internacional, 2017).

Un elemento que alimentó las dudas de la población sobre la salud del medio ambiente fue un estudio ambiental participativo que se realizó antes de la explosión del conflicto, por iniciativa de las organizaciones sociales locales, donde se confirmaba la existencia de altos niveles de contaminación (Humpel, 2011). Este estudio, por no ser auditado y fiscalizado por el Estado, fue rechazado por la compañía y autoridades de niveles altos del gobierno, creando mayor tensión y frustración en la población y el municipio.

La existencia de deficiencias en la gobernanza económica, social y ambiental de las industrias extractivas como las vistas en Espinar ha sido un incentivo importante para la aparición de mecanismos de participación ciudadana (Altomonte y Sánchez, 2015).

\footnotetext{
1 La Defensoría del Pueblo proporciona distintos recursos para conocer más sobre la conflictividad social del país. Por ejemplo, los reportes mensuales (ver https://www.defensoria.gob.pe/areas_tematicas/ paz-social-y-prevencion-de-conflictos/)yun mapainteractivo(verhttps://www.google.com/maps/d/u/0/ viewer?mid=1UZrUO9_x0ogNmcqrDvczGEtzfCc04g64\&ll=-10.147510082066333\%2C$75.205175 \& \mathrm{z}=6)$
} 
La participación ciudadana en América Latina fue diseñada como un mecanismo de gobernanza desde las bases sociales, con el fin de mejorar el desempeño de los gobiernos (Dagnino, Olivera y Panfichi, 2008). Este tipo de gobernanza puede ser entendido como un sistema de toma de decisiones desde el cual la sociedad civil ha ganado capacidad de influencia y decisión (Garrido-Vergara, Valderrama y Ríos, 2016). De esta forma, espacios participativos buscan superar asimetrías entre los funcionarios públicos y la sociedad civil para la toma de decisiones. En la actualidad, en países tanto desarrollados como en vías de desarrollo, se ha reconocido la utilidad y el valor de esta incorporación ${ }^{2}$.

En el caso de Espinar, el mecanismo participativo instaurado tras el conflicto socioambiental fue una mesa de diálogo. En el Perú, las mesas de diálogo eran entendidas inicialmente como una oportunidad para establecer la negociación directa frente a actores desinformados: ellas se creaban de modo posterior a la explosión de conflictos y los gobiernos actuaban de modo reactivo y violento reprimiendo las protestas (Paredes y De la Puente, 2014). Con el paso de los años, y particularmente desde el año 2012, el Estado reconoció formalmente la necesidad de incorporar el aporte de la sociedad civil local para mejorar la gobernanza de las industrias extractivas, adoptando posturas preventivas y transformadoras (Oficina Nacional de Diálogo y Sostenibilidad, 2014).

En el proceso de regular y promover mesas de diálogo como instancias de participación ciudadana, la experiencia de Espinar se reconoce desde el Estado como un momento clave de aprendizaje que ha inspirado ejercicios posteriores (Willaqniki, 2014). Esta mesa se entiende como exitosa, pues se logró aprobar nuevas medidas de fiscalización ambiental a través del involucramiento de la población (Banco Mundial, 2015), además de acordar nuevas formas de distribuir localmente la renta minera.

El presente artículo tiene por objetivo explorar las características de la dinámica de la mesa de diálogo en Espinar, y particularmente su grupo de trabajo medioambiental $^{3}$. Como se detallará más adelante, en el caso estudiado aparecieron barreras durante el proceso de participación que mermaron las reales capacidades de la sociedad civil de formar parte de la toma de decisión. Este análisis dialoga con la literatura que considera cómo la creación de espacios de participación ciudadana no garantiza, por su mera existencia, una mejora de la gobernanza de los territorios y la sociedad (Dagnino, 2008; Fischer, 2012). En este cuerpo de estudio, la participación puede ser entendida como un espacio donde los diferentes actores pugnan por mayor poder $y$, en este proceso, pueden reproducirse ejercicios de dominación

\footnotetext{
2 Para conocer más sobre la historia de la participación ciudadana, revisar Baiocchi y Ganuza (2014), Altschuler y Corrales (2013), Peters (2012), Fischer (2012) y Fung y Wright (2001).

3 Los otros dos grupos de trabajo fueron sobre desarrollo y responsabilidad social.
} 
(Gaventa, 2004; Svampa, 2008; Clarke, Coll, Dagnino y Neveu, 2014). Así, instrumentos como las mesas de diálogo necesariamente son conformadas por actores con distintos intereses y capacidades, expresando dinámicas conflictivas antes que puramente colaborativas o neutras (Fung y Wright, 2001).

Sin embargo, no todo proceso participativo implica dominación y fracaso. Un factor fundamental en el éxito de estos procesos es el esfuerzo por establecer mecanismos idóneos para todas y todos los participantes (Kothari y Cooke, 2001). De no tener estas metodologías y objetivos del proceso participativo como una oportunidad de transformación, pueden producirse resultados paradójicos de involucramiento ciudadano.

La presente investigación ha sido realizada a partir de un estudio de caso exploratorio. Las visitas a la provincia de Espinar se realizaron en octubre de $2013^{4}$. Se hicieron quince entrevistas semiestructuradas a actores clave: seis a funcionarios públicos del gobierno central y local, cuatro a dirigentes sociales y campesinos, cuatro a miembros de ONG y una a un representante de la compañía minera5.

En lo que sigue, se inicia con una descripción de la dinámica de la mesa de diálogo y sus resultados, y luego se analizan posibles causas subyacentes a la falta de acuerdos y a la frustración de la población participante. Esta reflexión prioriza las características de la regulación interna de la mesa y los retos políticos que genera para los funcionarios públicos peruanos tener una economía altamente dependiente de la actividad minera y, al mismo tiempo, responder a los cuestionamientos locales sobre la salud de las personas y el medio ambiente. En la última sección se presentan algunos aprendizajes que, se espera, sirvan para revisar el horizonte desde los cuales se imaginan los alcances de ejercicios de participación ciudadana.

\section{LA MESA DE DIÁLOGO EN ESPINAR}

La mesa de diálogo en Espinar contó con diversos recursos humanos y materiales que la posicionan como un caso emblemático de diálogo en el país (Willaqniki, 2014). Primordialmente, existió gran disposición de los actores involucrados por sacar adelante el proceso. Los representantes del gobierno provenían de altos niveles de toma de decisión y acudieron decenas de expertos y funcionarios de los sectores involucrados. Además, a diferencia de otros escenarios luego del conflicto social, la población de Espinar y sus representantes no estaban rechazando la presencia minera.

\footnotetext{
4 La autora realizó el campo en el marco del proyecto Participación Política Indígena en Espacios Locales. Este proyecto fue dirigido por Maritza Paredes (PUCP) y auspiciado por el Jurado Nacional de Elecciones e Idea Internacional.

5 El trato de los testimonios en el presente texto es anónimo. Si se desea conocer más sobre las entrevistas, contactarse con la autora a lorena.delapuenteburlando@gmail.com.
} 
En realidad, se trató de un escenario de conflicto que buscaba negociar la «convivencia» con la actividad 6 . Considerando que en Espinar se cuenta con una importante tradición de representación política local (Paredes, 2015), el perfil de la sociedad civil involucrada no podía ser más favorable: la dirigencia era experimentada y se deseaba la permanencia de la minería.

La mesa de diálogo en Espinar tuvo una larga duración y en ella participaron muchas y diversas personas. Se realizaron setenta reuniones y conjuntamente se involucró a 170 funcionarios públicos de los tres niveles de gobierno, treinta miembros de la compañía minera, cuarenta representantes de organizaciones de la sociedad civil y cerca de cien miembros de comunidades campesinas. Este último punto es importante, considerando que en Espinar existen 66 comunidades. De ellas, veinticinco participaron en la mesa de diálogo. También estuvieron presentes las rondas campesinas y los comités de regantes (Zeisser, 2015).

Como muestra el gráfico 1 , los representantes campesinos, junto con los dirigentes sociales, significaron cerca del $40 \%$ de participantes. El gobierno (nacional, regional y municipal) alcanzó el $46 \%$. De estos últimos, la mayoría representó al sector ambiental, destacando la importancia de este elemento 7 . La compañía minera tuvo una presencia menor de $10 \%{ }^{8}$.

\section{Gráfico 1. Perfil de los participantes de la MDE}

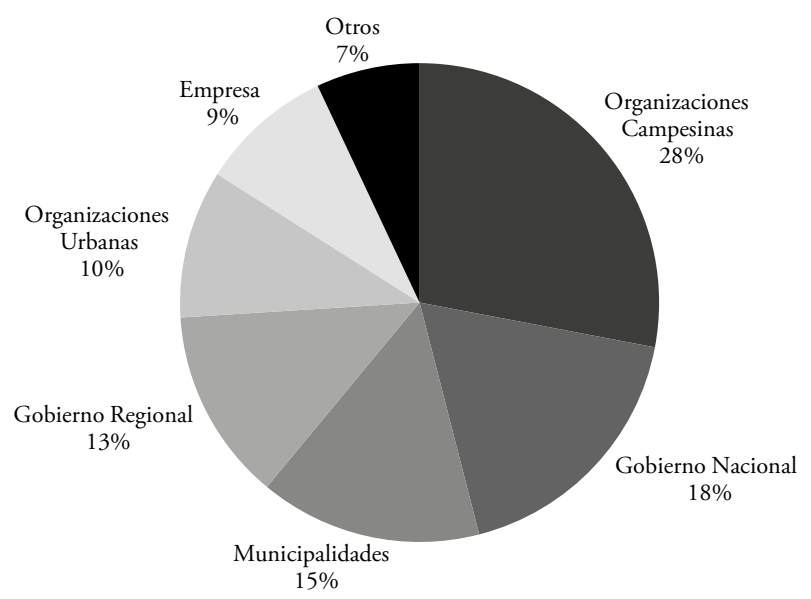

Fuente: Zeisser, 2015.

\footnotetext{
6 Categoría de José De Echave. Ver declaraciones en PERUMIN, 32 (2015): https://www.convencionminera.com/perumin32/index.php/prensa/407-nota-de-prensa-228

7 Incluso se definió que el ministro del Ambiente sería el representante del Estado nacional en la Presidencia Colegiada, la entidad más poderosa dentro de la mesa de diálogo de Espinar.

8 A diferencia de lo sucedido a inicios de 2000 (De Echave, Keenan, Romero y Tapia, 2009), en esta ocasión el diálogo fundamentalmente se realizó entre la sociedad civil y el Estado.
} 
Además de que la Mesa de Diálogo haya tenido una proporción importante de la sociedad civil urbana y rural presente, también estuvo formalmente involucrada en los tres grupos de trabajo (medio ambiente, responsabilidad social y desarrollo y producción), como muestra el cuadro $1^{9}$.

Cuadro 1. Miembros de la mesa de diálogo de Espinar (2012-2013)

\begin{tabular}{|c|c|c|c|}
\hline Instancia & Nacional & Regional & Local \\
\hline $\begin{array}{l}\text { Presidencia } \\
\text { colegiada }\end{array}$ & Ministro Minam & Presidente Gorecus & Alcalde MPE \\
\hline $\begin{array}{l}\text { Secretaría } \\
\text { técnica }\end{array}$ & Minam, Minem & Gorecus & MPE, Fudie, Xstrata S.A. \\
\hline \multicolumn{4}{|l|}{ Grupos de trabajo } \\
\hline Medio ambiente & $\begin{array}{l}\text { Minam (coord.) } \\
\text { OAAS, OEFA, } \\
\text { Ingemmet, Minsa, } \\
\text { DGCA, Digesa, ANA }\end{array}$ & $\begin{array}{l}\text { Gerencia de Recursos } \\
\text { Naturales y Medio } \\
\text { Ambiente } \\
\text { Diresa }\end{array}$ & \multirow{3}{*}{$\begin{array}{l}\text { Estado local y Estado } \\
\text { central (ámbito local), } \\
\text { MPE, municipalidades } \\
\text { distritales, Minagri, Minsa, } \\
\text { Minedu, Defensoría del } \\
\text { Pueblo } \\
\text { Organizaciones campesinas: } \\
\text { Fucae, comunidades } \\
\text { campesinas } \\
\text { Organizaciones urbanas: } \\
\text { FUDIE, FUJEK, gremios } \\
\text { ONG: CooperAcción, } \\
\text { Derechos Humanos sin } \\
\text { Fronteras. } \\
\text { Empresa: fundación Tintaya }\end{array}$} \\
\hline $\begin{array}{l}\text { Responsabilidad } \\
\text { social }\end{array}$ & $\begin{array}{l}\text { Minem (coord.) } \\
\text { OGGS, Minam }\end{array}$ & $\begin{array}{l}\text { Gerencia de } \\
\text { Desarrollo Social, } \\
\text { Subgerencia de } \\
\text { Comunidades } \\
\text { Andinas Amazónicas }\end{array}$ & \\
\hline $\begin{array}{l}\text { Desarrollo y } \\
\text { producción }\end{array}$ & $\begin{array}{l}\text { Minagri, DGCA } \\
\text { (coord.), ANA, PSI, } \\
\text { MVCS, Senasa }\end{array}$ & $\begin{array}{l}\text { Gerencia de } \\
\text { Desarrollo Económico } \\
\text { (coord.) }\end{array}$ & \\
\hline
\end{tabular}

Fuente: Zeisser (2015).

El nivel más alto de gobierno de la mesa, la Presidencia Colegiada, incluyó a autoridades nacionales, regionales y locales. El segundo rango más alto, la Secretaría Técnica, contó con el Frente Único de Defensa de los Intereses de Espinar ${ }^{10}$.

9 El grupo de trabajo sobre medio ambiente, objeto de investigación de este ensayo, estuvo coordinado por el Ministerio del Medio Ambiente y diferentes entidades intersectoriales para realizar monitoreos ambientales participativos.

10 El Frente de Defensa representa a distintas organizaciones principalmente urbanas, como la Asociación de Urbanizaciones Populares y Pueblos Jóvenes de Espinar, el Sindicato Único de Comerciantes del Mercado Central de Espinar y el Frente Único de Transportistas y Choferes de Espinar. Sin embargo, el rol de resistencia del Fudie durante el conflicto tradicionalmente ha sido central y disputa la representación de la provincia con las organizaciones campesinas. 
Las actividades del grupo de trabajo de medio ambiente fueron diversas. Entre ellas, destaca el desarrollo de un nuevo monitoreo ambiental que compense aquellos que se habían hecho con anterioridad y genere consenso ${ }^{11}$. Los reclamos ambientales en la provincia no son nuevos y han formado parte de las demandas sociales desde inicios de 2000, por lo cual se ha ido registrando información ambiental. Entidades como Osinergmin, Censopas y el propio OEFA, junto con las compañías titulares de la mina Tintaya, realizaron diversos monitoreos ambientales (algunos participativos) a lo largo de los años (Chávez, 2013; Rojas y Leiva, 2012). En el año 2002 se creó un Comité de Vigilancia Ambiental, planificando monitoreos ambientales periódicos. Posteriormente, en 2005 se acordaron compromisos en esta línea, como instalar un Comité de Vigilancia Ambiental Conjunto (Banco_Mundial, 2015).

El proceso de diálogo duró un ańo y medio, y a la luz de la cantidad y diversidad de personas involucradas, la mesa podría categorizarse como ampliamente participativa e incluyente. Sin embargo, con el transcurso del tiempo, empezó a aparecer una serie de dificultades que mermaron la capacidad de la sociedad civil de mantener el ritmo de las reuniones y la calidad de su representación.

En primer lugar, existió una sobrecarga en los dirigentes sociales urbanos que consistió en la centralización de roles en pocas personas, particularmente sobre los representantes del Frente de Defensa. El mandato general de la mesa era que cada organización debía elegir a sus representantes, pero el Frente no tenía muchos miembros entre los cuales optar: según los entrevistados, solo había cuatro dirigentes en capacidad de participar: el presidente, el vicepresidente, el secretario de prensa y el secretario de medio ambiente.

A pesar de que eran cuatro participantes, con el paso de los meses el secretario medioambiental no solo participó en el grupo de trabajo que le competía, sino que también tuvo que asumir el resto de puestos. Como él mismo explica: «En cada uno [de los grupos de trabajo] designaron a miembros de Frente de Defensa, pero [ellos] abandonaron los cargos porque el trabajo era diario, permanente [...]. Como yo era parte de la Secretaría Técnica, asumí los tres [cargos para cada subgrupo]». Esta representación no solo se enfrentaba a la alta demanda de tiempo sino también a un ambiente hostil postconflicto, pues varios dirigentes estaban siendo procesados por el presunto delito de incitar a la violencia en las protestas de $2012^{12}$.

11 Revisar Seizzer (2015, p. 36) para mayor detalle.

12 Un elemento a ser considerado es la criminalización de la protesta social (Instituto de Defensa Legal, 2012). En el Perú, desde hace varios años el control de conflictos sociales se ha caracterizado por una sistemática criminalización, donde dirigentes y participantes de protestas son agredidos, asesinados o procesados judicialmente. En Espinar, la historia no fue distinta y varios dirigentes (incluyendo al propio alcalde provincial) enfrentaron procesos judiciales mientras participaban en la mesa de diálogo. Con el tiempo, estos cargos no proceden, pues en la mayoría de los casos solo se trataba del derecho ciudadano a la protesta. 
En segundo lugar, hubo una subrepresentación de la población campesina, pues los participantes debieron rotar constantemente para cubrir la demanda de participación. A diferencia del caso de la representación social urbana con el Frente de Defensa, los representantes de la población campesina no podían sostener una participación constante. Esto se debió a que las y los dirigentes campesinos poseen aún más dificultades logísticas y de recursos para invertir tiempo en un proceso de diálogo que el resto de miembros. El presidente de la Federación de Campesinos explica: «Pensamos que [la mesa] iba a durar tres meses, pero se alargó bastante y nos llevó al cansancio. Muchos dirigentes no contamos con suficientes recursos económicos [para asistir a las reuniones]». La rotación de la dirigencia campesina es comprensible, pues participar en reuniones significaba dejar sus actividades productivas y una estrategia fue compartir la representación para aliviar la carga de trabajo y responsabilidades. Sin embargo, este hecho mermó la coordinación entre las organizaciones sociales y sus bases, disminuyendo la posibilidad de una participación más efectiva.

El gráfico 2 muestra la evolución de los asistentes a las sesiones del grupo de medio ambiente. Se puede apreciar cómo existe una tendencia a la baja en la cantidad de participantes a medida que se extiende el número de sesiones, lo que respalda las afirmaciones en torno al rol que jugó la duración de dieciocho meses de la mesa y lo difícil que es mantener el mismo grupo de trabajo durante todo el proceso.

Gráfico 2. Sesiones y asistentes al grupo de medio ambiente (2012-2013)

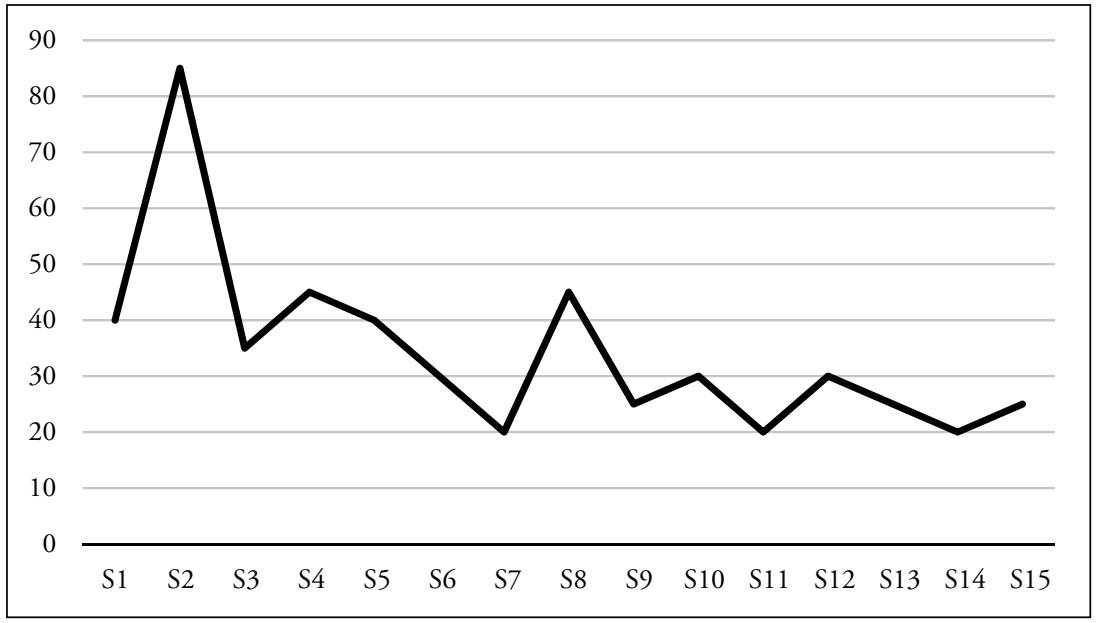

Fuente: Zeisser, 2015.

En este proceso, se registran capacidades distintas de participación entre funcionarios públicos y sociedad civil. Mientras que para los primeros participar era parte de su trabajo y se seleccionó a funcionarios de distintas oficinas especializadas para 
cada grupo, para los representantes locales fue un esfuerzo individual sin recursos económicos destinados a reconocer su logística ni las horas de trabajo perdidas. Un miembro de la empresa minera confirmó que «los mismos representantes de la sociedad civil eran los que participaban de todos los grupos: era una dinámica de mucha sobrecarga».

Un tercer y fundamental elemento que diferenció la calidad de la participación de la sociedad civil de la del gobierno fue el uso de un lenguaje técnico para la toma de decisión sobre el medio ambiente. En las entrevistas realizadas, la sociedad civil sintió que no pudo hacer oír su voz de igual forma que el gobierno. Un dirigente explicaba: «Cada sector cumple su función, su rol. La parte social no toca el tema técnico. Cumplimos en hacer llegar las quejas [...] con eso colaboramos los dirigentes». Sin embargo, los representantes civiles se vieron en situaciones en las cuales votaban a favor de temas que no necesariamente comprendían en toda su dimensión. La representante de la Vicaría, institución que apoyó a la población en el proceso, señaló que este punto puso a los dirigentes en desventaja: «Se puede manejar el lenguaje político, pero cuando llegaron a las mesas, ese lenguaje ya no servía [...] al momento [ellos] no tenían las capacidades de negociar porque el Estado imponía [el lenguaje]». Esta percepción fue compartida por el representante de la compañía entrevistado, quien afirmó que «en el momento de lucha política [los representantes sociales] tienen las herramientas, pero cuando te sientas en una mesa para discutir temas económicos y de producción, como que la política no alcanza».

Si bien hubo respaldo de algunos asesores provistos por ONG y la Iglesia católica, estos no estuvieron (ni debían estar) presentes en cada toma de decisión. En la práctica, se operó bajo un sistema de asesorías y discusiones previas, pero era muy limitado lo que se podía hacer, pues quienes se sentaban largas horas a discutir en la mesa eran representantes de otro tipo de saberes y preocupaciones. La misma representante de la Vicaría afirmaba: «Ha sido un diálogo vertical. [Los dirigentes] dicen que no han logrado entender todo. La mesa terminó en una discusión técnica. [Los asesores] insistimos en que el lenguaje se bajara, pero el Estado no quiere. Se cree que se baja el nivel. El riesgo es que el diálogo se hace con los expertos y el diálogo con la población se invisibiliza».

Como resultado de la poca calidad de la representación social, una vez culminado el proceso de diálogo hubo radicales discrepancias en torno a los resultados del monitoreo ambiental sobre la contaminación. Mientras el Estado aseguraba que solo existían menos de 3\% de puntos críticos de contaminación, la sociedad civil argumentó que estos sobrepasaban el 50\% de los puntos estudiados, tanto de aire y agua como de tierra, colocando en serio riesgo la salud de las personas (Zeisser, 2015). En estos puntos se habían identificado metales pesados, como mercurio, 
arsénico, cadmio y plomo. Así, los entrevistados afirmaban que la interpretación estatal de los resultados de los estudios había sido parcial y hasta errónea. Un miembro de la municipalidad provincial encargado de la gestión ambiental afirmó que «fue muy difícil colocar la palabra "contaminación” en el acta final, ni siquiera en el Informe Final [...]; hasta ahora la empresa se niega a aceptar contaminación, ella [y el Estado] habla [solo] de resultados anómalos».

Paradójicamente, la información del monitoreo ambiental no podía ser definitiva, porque era imposible contrastarla con información histórica sobre el comportamiento del medio ambiente. Ya se ha mencionado cómo existieron diversos esfuerzos, entre públicos y privados, por registrar los cambios del medio ambiente en el área de influencia minera en Espinar. Sin embargo, las distintas entidades encargadas de realizar estudios no presentaron continuidad en los lugares de muestreo, los momentos del año para realizarlos ni los procesos de participación a mediano y largo plazo. Así, llegado el año 2013 era difícil establecer causalidades, pues no se habían estudiado los mismos puntos de monitoreo en condiciones similares (Chávez, 2013; Rojas y Leiva, 2012). Además de esto, no existía información histórica registrada en la provincia. Este tipo de falencias en el seguimiento del medio ambiente no es exclusivo de Espinar: se trata de un problema nacional ${ }^{13}$.

De esta forma, considerando la diversidad de la información disponible, si bien el estudio de 2012 producto de la mesa de diálogo era de mayor y mejor calidad que los anteriores, no bastaba para establecer el origen y la antigüedad de la contaminación. Por poseer un carácter descriptivo, eran posibles distintas interpretaciones.

A pesar del factor ambiguo inherente al estudio y las grandes discrepancias entre el gobierno y la sociedad civil asesorada, en el acta final de la mesa el Estado afirmó que las condiciones de riesgo al ambiente eran solo moderadas, lo que generó el rechazo de la población (Secretaría Técnica de la Mesa de Diálogo de Espinar, 2013).

Los dirigentes sociales no respaldaron las conclusiones ambientales y trataron hasta el último momento de cambiar la interpretación a través de observaciones, pero estas fueron rechazadas. El Ministerio del Ambiente, la autoridad que presidía la mesa de diálogo, no aceptó las críticas e instó a la sociedad civil a «seguir caminando hacia el cierre concertado de la mesa» (Zeisser, 2015). Posteriormente, en una reunión en Lima del equipo técnico interinstituciona ${ }^{14}$ se concluyó que el documento de la sociedad civil, antes que un pedido de aclaraciones, era una "propuesta de cambio de sentido de las conclusiones finales del Informe». Se indicó que el Informe Final ya había sido presentado, validado y suscrito en su momento

13 En una entrevista con Ricardo Giesecke, exministro del Ambiente, este explicaba cómo la data disponible es sumamente escasa, lo cual dificulta las capacidades de monitoreo y evaluación.

14 Conformado por representantes del sector salud, energía y minas y ambiente: Minam, ANA, Digesa, Oefa, Ingemmet, Senasa y Censopas. 
por los miembros durante el desarrollo de la mesa (Zeisser, 2015), entonces no había mayor discusión o revisión que hacer. Por su parte, la compañía minera emitió un comunicado donde afirmaba que la contaminación no era por sus operaciones, sino que se trataba de una característica propia del lugar (La Mula, 2013; El Comercio, 2014). Este punto de vista fue corroborado por el representante de la empresa entrevistado: «Se ha demostrado [con el monitoreo participativo] que no hay contaminación, dieciséis instituciones del Estado han participado en una evaluación reciente [...], en realidad es una contaminación natural del subsuelo».

La situación generó una gran frustración y rechazo. La sociedad civil no comprendía por qué, si había cierta evidencia que señalaba contaminación y había más de un modo de interpretarla, se decidía entenderla como de carácter leve. Un dirigente nos decía: «Nos han ganado bastante espacio [...] los demás [dirigentes] no sabían, por ahí que nos ganaron [...]. Para una discusión técnico-científica se necesita experiencia de carrera [profesional]. La discusión era sobre uso y manejo de laboratorios, interpretación de análisis. Era frustrante».

\section{DisEŃO Y DILEMAS POLÍTICOS}

La falta de acuerdos en la temática ambiental y la frustración de la sociedad civil llama la atención sobre la dinámica de la mesa. Vistos los resultados y testimonios, la mesa no creó canales suficientes para incorporar los saberes locales en los debates políticos en torno a la condición socioambiental de la provincia.

La mesa de diálogo tuvo como objetivo impulsar «acciones integradas y coordinadas que permitan atender y buscar alternativas de solución a la problemática socioambiental $»^{15}$. Pero, considerando los testimonios de la sociedad civil, la concreción de aquellas "acciones integradas" tuvo límites en definir y permitir con mayor claridad cómo se iba a realizar la toma de decisión. Esto tuvo un particular impacto en el grupo de trabajo del medio ambiente debido a su carácter técnico y a la ausencia de información suficiente para interpretar los resultados del monitoreo ambiental conjunto.

Un primer elemento a evaluar es la metodología aplicada para la toma de acuerdos. Este método para la toma de decisión se aprobó al momento de su creación, pero tuvo un carácter general. La Resolución Ministerial que fundó la mesa solo regulaba la distribución de funciones y el tiempo (inicial) de funcionamiento de la mesa. El pacto entre los participantes fue que los acuerdos se tomarían por mayoría (presente), las discusiones serían privadas y las decisiones se pondrían a disposición para que fuesen aprobadas por las entidades superiores de la mesa (Zeisser, 2015).

15 RM 164-2012-PCM. 
Pero varios elementos de la dinámica no fueron previstos en esta metodología. Por ejemplo, qué hacer ante la falta de consistencia de algunos miembros (caso de representantes campesinos), cómo definir el cierre de temas de alto contenido técnico ante participantes muchos con pocos niveles de instrucción o cómo validar los conocimientos locales ante la ausencia de información histórica suficiente.

Lo visto en Espinar, sin embargo, no es sorprendente considerando el marco institucional que regula los procesos de diálogo: a la fecha no existe una regulación detallada. En realidad, se espera que las experiencias participativas se regulen por mandatos generales (Maldonado, 2013). Las normas actuales piden que los espacios de diálogo se autorregulen. La razón es permitir que cada iniciativa sea capaz de adaptarse a las características del grupo humano que conforma la mesa y al contexto sobre el cual se construye el diálogo (Willaqniki, 2013). En las primeras iniciativas para regular la participación ciudadana en el sector extractivo (2008), se solicitaba que las metodologías fuesen definidas por «autoridades competentes» que establecerían «mecanismos formales» o "protocolos de relacionamiento» para cada escenario, pero no se proveía de guías metodológicas de implementación reconocidas a rango de ley ${ }^{16}$. Esta perspectiva abierta se mantiene hasta hoy, pues las normas fundacionales de la ex Oficina Nacional de Diálogo y Sostenibilidad, hoy Secretaría de Gestión Social y Diálogo, tampoco tienen la preocupación por detallar metodologías.

Las preguntas que se abren son los riesgos de seguir este tipo de lógicas en contextos sociales donde existen asimetrías importantes entre la sociedad civil, funcionarios públicos y representantes de grandes compañías mineras. Pensemos en asimetrías en torno a niveles educativos, de clase y de género, además de las fuertes diferencias culturales.

Los espacios de participación ciudadana se crearon para que la sociedad civil y sus intereses fuesen incorporados en la toma de decisión pública. En este sentido, distintos saberes e intereses deberían ser incorporados. Así, no es necesario que la población tenga conocimientos técnicos para participar de un espacio de diálogo: su presencia es necesaria para recoger distintas perspectivas en la búsqueda por mejorar la gestión pública (Fischer, 2012). De no considerar estas diferencias, se establecen escenarios de exclusión antes que de inclusión.

«Fue una discusión más técnica que social [...]. Siento que no estuvimos a la altura de la situación. Nos faltó un peso técnico. Te traen a los mejores [especialistas]. Creo que [los dirigentes] debieron tener un perfil más alto desde un inicio» fue el balance de un antiguo dirigente del Frente de Defensa. En su testimonio se detecta la frustración de no sentir suficiente control sobre el proceso. Esta inconformidad

16 Ley 28611, DS 028-2008-EM. 
con la experiencia en la mesa de diálogo expresa no solo la reproducción de asimetrías ante la falta de metodologías más incluyentes, sino que, paradójicamente, este carácter excluyente se fue construyendo durante la cotidianeidad de la participación y no fue evidente para los participantes.

La sociedad civil nunca "pateó el tablero». A pesar de las dificultades, no se rompió el diálogo. Los representantes entrevistados señalaron que confiaban en que participar iba a ser útil para sus demandas y de facto y que sí fueron incluidos. La población y sus representantes ayudaron a definir los puntos de monitoreo ambiental y acompañaron en el recojo de las muestras. También hubo representantes en cada reunión donde se discutió el origen y magnitud de la contaminación. En este ejercicio, acordado por todas las partes, no era sencillo distinguir los límites de su involucramiento. Como recuerdan, todos los participantes deseaban el diálogo y reconocían la relevancia política de no dejar de asistir, pero, dadas las condiciones, el resultado terminó siendo frustrante y sorpresivo.

Por un lado, el Estado llenó los cupos de los participantes con expertos en asuntos socioambientales que contaban con recursos y tiempo para participar de la mesa, pero por otro, se sentó una sociedad civil que no tenía las mismas capacidades de participar, no porque sus conocimientos o preocupaciones no fueran legítimos, sino porque los métodos de diálogo no fueron adaptados a sus necesidades particulares para hacer oír su voz. Si bien se promovió que tuviesen presencia y voto, no se evaluó la sobrecarga para las y los dirigentes, la pesada demanda para la población campesina ni tampoco los saberes no científicos para ponderar impactos que no podían conocerse bajo la luz de monitoreos técnicos disponibles.

Idealmente, la metodología debería estar diseñada para que los procesos de toma de decisión sean suficientes para resguardar la capacidad de participación influyente de la sociedad civil (Maldonado, 2013) ${ }^{17}$. Quiénes deciden, cómo se discute, qué condiciones deben existir para poder llegar a acuerdos, cuáles son suficientes para suspender discusiones, entre otros puntos fundamentales, son las preguntas que la metodología podría ayudar a responder (Defensoría del Pueblo, 2017). Si bien la apuesta por la flexibilidad y la adaptación del gobierno peruano tiene objetivos en favor del diálogo, en contextos como los vistos en Espinar puede operar en sentido contrario, reproduciendo asimetrías entre los participantes.

En respuesta a estos escenarios, en un informe de 2016 la Presidencia del Consejo de Ministros ha reconocido la necesidad urgente de aprobar normativas más idóneas para posibilitar una efectiva participación. Así, superar el desafío de la efectividad

\footnotetext{
17 Una comparación interesante es la metodología «intercultural» que regularía la consulta previa en el Perú. Para una reflexión sobre la aplicabilidad actual de metodologías interculturales, revisar Sanborn, Hurtado y Ramírez (2016).
} 
de los procesos se logrará cuando se construya la arquitectura necesaria a través de normas, protocolos, estrategias y metodologías comunes a varios niveles de gobierno que potencien sus instituciones (ONDS, 2016). Este diseño es aún una tarea pendiente y los constantes cambios en la entidad pública encargada de regular estos procesos parecen no contribuir a aclarar los instrumentos ${ }^{18}$.

La discusión en torno a las metodologías que regulan los procesos de diálogo debe también considerar desde dónde se diseñan y se promueven mecanismos participativos. Es decir, ¿cuál es el horizonte de los actores estatales que se ven obligados a evaluar proyectos mineros tras conflictos locales? ¿Hasta dónde se imagina el nivel de influencia de la sociedad civil? ${ }^{19}$.

Un gobierno puede poseer un interés genuino por escuchar a la población local en espacios participativos, pero aceptar las agendas de la sociedad civil como mandatos de gobernanza en el mismo rango que aquellas agendas que promueven la extracción es un paso grande para países con economías (Baca, 2017) y sistemas políticos (Durand y Crabtree, 2017) dependientes de sostener tales niveles productivos como el Perú.

Nuestro país posee una larga historia de dependencia económica hacia la exportación de materias primas, dependencia que ha marcado su desarrollo institucional público (Dargent, Orihuela, Paredes y Ulfe, 2017). En esta historia, las élites políticas y económicas han jugado un rol central en reproducir cómo entender y promover las industrias extractivas, particularmente la minería (Durand y Crabtree, 2017).

La gobernanza de la minería del Estado peruano suele ser caracterizada como parte de las lógicas «extractivistas» de la región (Gudynas, 2016) ${ }^{20}$. El extractivismo es la creencia compartida entre los funcionarios públicos encargados de sectores mineros y energéticos y aquellos vinculados con las industrias extractivas (economía, transportes, construcción, etc.) sobre la necesidad de preservar dinámicas altas de producción. Muchas veces se comporta como un «imperativo» a causa de la tradición extractiva del país (Arsel, Hogenboom y Pellegrini, 2016). Esta creencia

18 La institucionalidad pública para regular procesos de diálogo ha sufrido cambios importantes en los últimos años. La Presidencia del Consejo de Ministros continúa siendo la entidad que aglomera las iniciativas, pero inicialmente se trató de una entidad autónoma con la Oficina Nacional de Diálogo y Sostenibilidad (creada en 2012). Pero en 2017, luego de la renuncia de su último director, se optó por crear el Viceministerio de la Gobernanza Territorial, y dentro de este Viceministerio se creó la Secretaría de Gestión Social y Diálogo. Revisar http://www.pcm.gob.pe/wp-content/uploads/2018/07/ ABC-de-la-SGSD.pdf

19 Es importante destacar que aquí nos estamos enfocando en procesos de diálogo postconflicto. Otra gran esfera de trabajo en mecanismos de diálogo son los espacios de prevención y desarrollo. Revisar la página del Viceministerio de Gobernanza Territorial para conocer más sobre estas iniciativas: http://www.pcm.gob.pe/entidades-pcm/

20 Para conocer más sobre la historia político-económica de la región latinoamericana y la minería, revisar Bulmer-Thomas (2007), Thorp (2012) Altomonte y Sánchez (2015), Haarstad (2012). 
prioriza la captura de rentas por sobre los costos ambientales y sociales (Ballón, Viale, Monge, Patzy y De la Puente, 2017), pues lo más importante es continuar la expansión de la frontera minera y petrolera para financiar al sector público (Svampa, 2016).

A escala regional, el extractivismo ha definido un desarrollo institucional particular que ha posicionado a los sectores vinculados con la minería en una esfera alta de poder e influencia por encima de otros sectores, incluyendo al sector medioambiental (De la Puente y Ballón, 2019) ${ }^{21}$. En este sentido, las promesas de la participación ciudadana como mecanismos de influencia sobre decisiones públicas para regular asuntos ambientales en contextos mineros pueden estar confrontadas con intereses vitales para las autoridades estatales, y estos intereses pueden guiar su acción durante procesos de diálogo.

En nuestro caso, la importancia política del proyecto de Espinar era muy alta. El proyecto minero en la provincia forma parte de uno de los principales ejes de producción en el país (CooperAcción, 2016). Desde la década de 1980, la mina Tintaya ha significado un proyecto importante tanto para la economía nacional como para la local. Solo en el año del conflicto estudiado, la mina Tintaya aportó con 37 millones de dólares en impuestos para el Estado peruano (Antapaccay, 2014). En Espinar también está presente el proyecto minero Antapaccay, que inició operaciones precisamente en 2012 y poseería alrededor de 720 millones de toneladas de cobre en concentrado por un año como reservas estimadas (Banco Mundial, 2015). Asimismo, gracias a un acuerdo con la municipalidad provincial vigente desde inicios de la década de 2000, la mina ha aportado directamente al municipio sumas millonarias de dinero. Desde que existen los aportes directos, esta suma significó 70 millones de dólares (Glencore, 2013). El Convenio Marco fue el instrumento innovador de gestión de la renta escogido, el cual fue resultado de los procesos de diálogo de inicios de la década de 2000 (Camacho y Lossio, 2007). Este consistió en un acuerdo directo entre la empresa y la sociedad civil para que aquella aporte voluntariamente un porcentaje (alrededor del tres por ciento) de sus ganancias al gobierno local (De Echave, 2005) 22 .

\footnotetext{
21 Para explorar más literatura en este aspecto, revisar Perreault (2005, 2013), Gobel y Ulloa (2016), Ballón et al. (2017), Gudynas (2017), Valencia (2017) y Temper, Walter, Rodriguez, Kothari y Turham (2018).

22 En el presente artículo se ha priorizado lo visto en el grupo de trabajo de medio ambiente, pero la demanda local por compartir más beneficios de la presencia minera fue un eje central que desencadenó el conflicto. La sociedad civil y el municipio demandaron un aumento de estos aportes, porque consideraban que la empresa minera había centralizado la gestión del dinero y había entonces una pugna por los montos y la distribución. Si bien la discusión sobre la renta y los aportes fue clave, resultó más sencillo alcanzar los acuerdos en torno a proyectos de desarrollo e inversión que los acuerdos ambientales.
} 
La relevancia macroeconómica de los proyectos mineros en Espinar no se reduce a lo realizado en la provincia, sino también a su posición estratégica en el sur andino (De Echave, 2015). La provincia de Espinar cumple un rol importante en la producción minera del sur del país gracias a su conexión con el proyecto minero de mayor inversión en la historia del Perú: Las Bambas (Gestión, 2016) ${ }^{23}$. Las Bambas aprovecha las instalaciones de la mina Tintaya y de Antapaccay para su producción, conexión que fue planificada por las compañías a cargo de los tres proyectos ${ }^{24}$.

Ante este escenario de fuertes compromisos e intereses económicos, el horizonte de la «no extracción» o continuar con la detención del proyecto no formaba parte de las expectativas del sector público. En la propia Acta Final de la Mesa se expresa esta tensión de intereses: «[...] teniendo en cuenta los diversos factores, nacionales e internacionales, relacionados con los precios de los productos minerales, costos de producción, política tributaria, inflación minera, productividad y estabilidad social, entre otros factores; las partes de la Mesa de Diálogo destacan la importancia del diálogo informado entre los actores locales, la empresa y los responsables de la política minera y tributaria, con la finalidad de mantener y/o ampliar las oportunidades para el desarrollo de Espinar correspondientes al año 2014 y siguientes» (Secretaría Técnica de la Mesa de Diálogo de Espinar, 2013, p. 4). Paradójicamente, detener la actividad minera tampoco formaba parte de las agendas de sociedad civil (por lo menos, no de la totalidad de los representantes): tradicionalmente la minería se entiende como una fuente de desarrollo local. La diferencia entre ambos actores estaba en reconocer responsabilidades, remediaciones ambientales y compensaciones a las personas y comunidades afectadas. Todas estas medidas en discusión habrían impactado sobre el funcionamiento regular de la mina, arriesgando los niveles de producción (y captación de renta).

Así, distintos actores tienen diversos puntos de partida cuando se sientan en una mesa de diálogo. Pero, como el caso ilustra, aquellos intereses y saberes que se alejan del impulso minero extractivista pueden tener menos capacidad de influir en la toma de decisión sobre las políticas que regulan la acción del sector. Lo llamativo del caso de Espinar es que la propia información ambiental disponible no era definitiva. La ausencia de información histórica sobre la zona generaba un escenario donde más voces (y fuentes de información) podrían haber jugado un rol más constructivo en comprender qué estaba sucediendo en el área de influencia minera.

\footnotetext{
23 Las Bambas inició operaciones en 2015 y en la actualidad cumple un rol central en la producción de cobre nacional, principal recurso de exportación minero del país en contextos de recesión y baja de precios internacionales (CooperAcción, 2016; Gestión, 2016).

24 La mina Tintaya ha pasado por diversos procesos de compraventa e incluso fusión de capitales, lo que dio origen al cuarto grupo minero más grande del momento precisamente en 2012 (Banco Mundial, 2015; Gestión, 2014).
} 
Este proceso ha tenido consecuencias sobre el tratamiento del medio ambiente, elemento que aún está en disputa entre la población y el gobierno. A pesar de que la mesa de diálogo formalizó la creación de una serie de monitoreos a futuro, posteriormente aparecieron serias acusaciones en torno al uso y transparencia de la información recolectada en estas iniciativas de monitoreo. Aparentemente, los niveles de contaminación en la sangre de la población local eran más altos y estaban afectados por más metales que aquellos que el gobierno comunicó inicialmente ${ }^{25}$.

\section{REFLEXIONES FINALES}

¿Cuáles son los aprendizajes de este caso? La mesa de diálogo en Espinar es expresión de los límites y también de las potencialidades futuras de procesos de diálogo en el Perú.

Fundamentalmente, la experiencia en Espinar enseña que la creación por sí misma de espacios participativos no es condición suficiente para la influencia o incorporación de saberes y expectativas civiles en tomas de decisión y, por el contrario, puede servir para reproducir asimetrías de poder (Gaventa, 2004).

El proceso de diálogo no aplicó suficientes instrumentos como para superar asimetrías entre los participantes. La sobrecarga a dirigentes sociales urbanos, la subrepresentación de intereses campesinos y el uso de un lenguaje técnico marginalizó una interpretación local del origen y magnitud de la contaminación. Estas características del proceso no permitieron, a la luz de los hechos y la percepción de la sociedad civil, un intercambio real de conocimientos que mejorase la toma de decisión.

Paradójicamente, estas asimetrías no son evidentes a primera vista, pues de distintas maneras la población está presente. Desde el inicio hasta su fin, la sociedad civil estuvo dispuesta a debatir y participó en iniciativas de fiscalización ambiental. Pero la decisión de utilizar procesos estandarizados que responden más a las lógicas propias del sector que a la realidad social local mermó las potencialidades del

25 Organizaciones de la sociedad civil y población local afirman que el Ministerio de Salud tomó muestras sanguíneas en 2010 a modo de control, pero solo años después se reconoció que los resultados completos no habían sido compartidos con la opinión pública ni con los evaluados. Precisamente, estos resultados demostraban aún más presencia de contaminación en la población (Amnistía Internacional, 2017). Revisar: https://www.amnistia.org.pe/noticia/peru-emergencia-sanitaria-contaminacion-aguaconsumo-humano-espinar/. ONG como el Instituto de Defensa Legal han asumido posiciones de litigio ante la emergencia sanitaria y han llegado a tener audiencias en la Comisión Interamericana de Derechos Humanos: http://www.justiciaviva.org.pe/new/wp-content/uploads/2018/06/ Art\%C3\%ADculo-La-contaminaci\%C3\%B3n-en-Espinar-por-metales-pesados-comienza-a-cobrarsus-primeras-v\%C3\%ADctimas.pdf. Adicionalmente, hacia fines de 2018 se inició una campaña por visibilizar el problema: http://cooperaccion.org.pe/espinar-por-una-entrega-de-resultados-dedosaje-con-interpretacion-y-orientaciones-claras/. Para conocer más, revisar el documental interactivo La vida no vale un cobre: https://lavidanovaleuncobre.com/\#INICIO 
diálogo, generando desacuerdos y frustración. En la actualidad, la contaminación del medio ambiente continúa marcando la agenda política de la provincia.

Ante los resultados de un proceso que concentró grandes recursos humanos y económicos, además de contar con el interés y apoyo de todos los agentes involucrados, vale la pena preguntarse qué se está institucionalizando como mecanismo de gobernanza tras conflictos mineros. ¿Es suficiente para revisar las políticas del sector y mejorarlas?

Lejos de tratarse de un fin maquiavélico del Estado, la reproducción de asimetrías a través de espacios de participación puede ser el resultado de buenas intenciones enmarcadas en discursos y prácticas institucionales contradictorias. Después de todo, los Estados actúan bajo los marcos que les parecen adecuados para su propia reproducción (Scott, 1998). En nuestro caso, la agenda extractivista era el punto de partida desde el cual los funcionarios públicos enmarcaron el proceso de diálogo que ellos mismos dirigían y pautaban. Así, se puede interpretar que el uso de un lenguaje especializado era un requisito fundamental a la vista de los funcionarios públicos, pues finalmente estamos hablando del medio ambiente, que suele ser medido aplicando estándares científicos (Vila, 2014).

Además de incorporar a más y mejores técnicos que estudiasen el ambiente con más y mejor tecnología, había que integrar a más personas para incrementar la transparencia. Así, se invitó a todas las organizaciones sociales y se permitió que las comunidades campesinas continuaran inscribiendo a más representantes. Todo fue, pues, racional. El resultado, sin embargo, fue la creación de barreras para incorporar saberes locales. Es decir, incluso para las autoridades públicas — quienes razonan desde sus propios horizontes de sentido-, la reproducción de asimetrías puede ser una consecuencia no buscada de su acción.

Continuar con este tipo de estrategias de diálogo en escenarios postconflicto da el mensaje de que la opinión local se incluye siempre y cuando esté en línea con las expectativas de los actores más poderosos en la negociación, resultado que puede ser opuesto a los intereses del Estado cuando promueve espacios participativos. Así, el resultado más grave es el agotamiento de los participantes más vulnerables. Un dirigente expresaba este sentimiento: «Lo que hemos palpado del desarrollo de la mesa es que ha habido una parcialización de la empresa con el gobierno. Eso ha quedado totalmente claro. Dos contra uno era esa mesa. A tal punto que los mediadores de los ministerios se han parcializado en favor de la minera. Cuando poníamos nuestra propuesta nos desestimaban, nos pedían que aceptáramos las propuestas mineras».

Cuando la sociedad civil en el Perú se involucra en situaciones de participación excluyente creyendo que ejerce influencia, puede volverse parte de un mecanismo que legitima la expansión de las actividades extractivas bajo criterios similares a los que generaron los conflictos ambientales en primer lugar. En el caso estudiado, al tener un acta final de una mesa de diálogo donde cientos de actores de la sociedad 
civil fueron involucrados, los futuros reclamos sobre la performance ambiental de la actividad minera pueden haber agotado las vías institucionales para hacerse escuchar. Incluso tales reclamos pueden ser entendidos como ilegítimos. Tomemos por ejemplo la lectura del representante de la empresa minera sobre las motivaciones de los dirigentes y su negativa a firmar los acuerdos: «Nuestra lectura [como compañía] es que se cambia el régimen municipal [en 2010] y la municipalidad provincial entra con otras ideas para renegociar [los aportes directos de la empresa minera a la municipalidad] y la única manera de lograr que la empresa se sentara a dialogar era con el tema ambiental». Hoy el Perú continúa concentrando altos niveles de conflictividad socioambiental, y preservar los mencionados instrumentos de participación que promueven la polarización podría hacer poco por prevenir futuros escenarios conflictivos.

¿Pero cómo transformar este escenario? No se trata, pues, de una discusión sobre «estar a la altura de la situación»: es el Estado peruano el principal responsable de desarrollar todos los canales necesarios para que tanto los saberes locales como los saberes técnicos contribuyan a mejorar actuales y futuras políticas de la gobernanza de la minería en el país. Después de todo, el fin de la participación ciudadana es superar brechas entre expectativas y resultados de políticas públicas; en nuestro caso, la calidad de la fiscalización y remediación ambiental. Considerando la falta de información histórica sobre el medio ambiente, la percepción y experiencia de los habitantes tradicionales del área de influencia podría cumplir un rol importante.

En un país donde se continúa apostando por un modelo productivo basado en la minería, la pregunta por el involucramiento efectivo de la población local en espacios de diálogo continúa abierta y con la tarea pendiente de mediar la convivencia sostenible entre la sociedad civil, el medio ambiente y las industrias extractivas en el Perú.

\section{REFERENCIAS}

Altomonte, H. y Sánchez, R. (2016). Hacia una nueva gobernanza de los recursos naturales en América Latina y el Caribe. Libros de la CEPAL, 139 (LC/G.2679-P). Santiago: Comisión Económica para América Latina y el Caribe (CEPAL). https://doi. org/10.18356/0edf6614-es

Altschuler, D. y Corrales, J. (2013). The promise of participation: Experiments in participatory governance in Honduras and Guatemala. Nueva York: Palgrave Macmillan. https://doi.org/10.1057/9781137271846

Amnistía Internacional (2017). Estado tóxico, violaciones del derecho a la salud de pueblos indígenas en Cuninico y Espinar, Perú. Londres: Amnistía Internacional.

Antapaccay (2014). Informe de sostenibilidad. Lima: Antapaccay. 
Arsel, M., Hogenboom, B. y Pellegrini, L. (2016). The extractive imperative in Latin America. The Extractive Industries and Society, 3(4), 880-887. https://doi.org/ 10.1016/j.exis.2016.10.014

Baca, E. (2017). La nueva agenda de la sociedad civil frente a las industrias extractivas en América Latina después del súper ciclo y la carrera hacia el fondo, reporte Perú. Lima: Grupo Propuesta Ciudadana, Natural Resource Governance Institute, Fundación Ford.

Baiocchi, G. y Ganuza, E. (2014). Participatory budgeting as if emancipation mattered. Politics \& Society, 42(1), 29-50. https://doi.org/10.1177/0032329213512978

Ballón, E., Viale, C., Monge, C., Patzy, F. y De la Puente, L. (2017). La agenda de la sociedad civil frente a las industrias extractivas en América Latina. Lima: Natural Resource Governance Institute, Fundación Ford.

Banco Mundial (2015). Construyendo desde el conflicto: las mesas de diálogo de Tintaya y Moquegua en Perú. Washington: Banco Mundial.

Bebbington, D.H. y Bebbington, A. (2012). Post-what? extractive industries, narratives of development, and socio-environmental disputes across the (ostensibly changing) Andean Region. En H. Haarstad (ed.), New political spaces in Latin American natural resource governance (pp. 17-37). Nueva York: Palgrave Macmillan US. https://doi.org/10.1057/9781137073723_2

Bulmer-Thomas, V. (2007). Globalization and the New Economic Model in Latin America. En V. Bulmer-Thomas, J. Coatsworth y R. Cortes (eds.), Cambridge Economic History of Latin America (¿pp -pp?). Nueva York: Cambridge University Press. https:// doi.org/10.1017/CHOL9780521812900

Camacho, L. y Lossio, F. (2007). La mesa de diálogo de la provincia de Espinar (Cusco): solucionando conflictos entre la sociedad civil local y la empresa minera BHP Billiton Tintaya sin la presencia del Estado. En A. Panfichi (ed.), Participación ciudadana en el Perú: disputas, confluencias y tensiones (pp. 147-229). Lima: PUCP.

Chávez, M. (2013). Informe: Análisis de los monitoreos ambientales realizados en zonas de influencia de las operaciones mineras de la unidad minera Tintaya. Lima: Oxfam.

Clarke, J., Coll, K., Dagnino, E. y Neveu, C. (2014). Disputing citizenship. Bristol, G.B.: Policy Press. https://doi.org/10.2307/j.ctt9qgzqg

CooperAcción (2016). Metales pesados tóxicos y salud pública: el caso de Espinar. Lima: Broederlijk Delen, CooperAcción, Derechos sin Fronteras, IDL.

Dagnino, E. (2008). Challenges to participation, citizenship and democracy: perverse confluence and displacement of meaning. En A.J. Bebbington, S. Hickey y D.C. Mitlin (eds.), Can NGOs make a difference? The challenge of development alternatives (pp. 55-70). Londres: Zed Books.

Dagnino, E., Olivera, A. y Panfichi, A. (2008). Innovación democrática en América Latina: una primera mirada al proyecto democrático-participativo. En C. Raventós, Innovación democrática en el sur, participación y representación en Asia, África y América Latina. Buenos Aires: Clacso. 
Dargent, E., J.C. Orihuela, J. Paredes y M.E. Ulfe (eds.) (2017). Resource Booms and Institutional Pathways: The Case of the Extractive Industry in Peru. Cham, Suiza: Palgrave MacMillan. https://doi.org/10.1007/978-3-319-53532-6

De Castro, F., Hogenboom, B. y Baud, M. (eds.). (2016). Environmental Governance in Latin America. Basingstoke y Nueva York: Palgrave MacMillan. https://doi. org/10.1007/978-1-137-50572-9

De Echave, J. (2015). Mineroducto del proyecto minero Las Bambas. Lima: CooperAcción.

De Echave, J., Keenan, K., Romero, M. y Tapia, A. (2005). Los procesos de diálogo y la administración de conflicto en territorios de comunidades: el caso de la mina Tintaya en el Perú. Lima: CooperAcción.

De la Puente, L. y Ballón, E. (2019). La gobernanza centralizada de la minería, el gas y el petróleo en los países andinos, ¿oportunidades de transformación? Lima: NRGI.

Defensoría del Pueblo (2017). El valor del diálogo. Serie Documentos defensoriales, documento $\mathrm{N}^{\circ} 29$.

Defensoría del Pueblo (2019). Reporte de Conflictos Sociales $N^{0} 178$. Adjuntía para la Prevención de Conflictos Sociales y la Gobernabilidad.

Deonandan, K. y Dougherty, M. L. (eds.).(eds.) (2016). Mining in Latin America: critical approaches to the new extraction. Nueva York y Londres: Routledge. https://doi. org/10.4324/9781315686226

DS 028-2008-EM. Reglamento de participación ciudadana en el subsector minero. Ministerio de Energía y Minas.

Durand, F. (2016). Cuando el poder extractivo captura al Estado. Lobbies, puertas giratorias y paquetazo ambiental en Perú. Lima: Oxfam.

Durant, F. y Crabtree, J. (2017). Perú: élites de poder y captura política. Lima: Universidad del Pacífico.

El Comercio (2012). Protestas en Cusco: pobladores de Espinar piden comisión de alto nivel. 24/5/2012.

El Comercio (2014). La OEFA impone una multa de S/. 235.600 a Xstrata Tintaya. 2/1/2014.

El Comercio (2018). Defensoría: 279 personas fallecieron en conflictos sociales. 22/10/18.

Fischer, F. (2012). Participatory Governance: From Theory to Practice. En L. David, The Oxford Handbook of Governance (¿pp-pp ?). Oxford: OUP. https://doi.org/ 10.1093/oxfordhb/9780199560530.013.0032

Flemmer, R. y Schilling-Vacaflor, A. (2016). Unfulfilled promises of the consultation approach: the limits to effective indigenous participation in Bolivia's and Peru's extractive industries. Third World Quarterly, 37(1), 172-188. https://doi.org/10.10 80/01436597.2015.1092867

Flores, C. (2016). Conviviendo con la minería en el sur andino. Experiencias de las mesas de diálogo y desarrollo de Espinar, Cotabambas y Chamaca. Lima: CooperAcción, Oxfam.

Foucault, M. (1980) Power/knowledge: Selected interviews and other writings, 1972-1977. Nueva York: Pantheon. 
Fung, A. y Wright, E. O. (2001). Deepening democracy: innovations in empowered participatory governance. Politics and society, 29(1), 5-42. https://doi.org/ $10.1177 / 0032329201029001002$

Garrido-Vergara, L., Valderrama, L.M. y Rios, J. (2016). Democracia deliberativa, instituciones y participación ciudadana en América Latina. Política, 54(2), 255-275.

Gaventa, J. (2004). Towards participatory governance: assessing the transformative possibilities. En S. Hickey y G. Mohan, Participation--from tyranny to transformation? Exploring new approaches to participation in development (pp. 25-41). Londres: Zed Books.

Gestión (2014). Glencore Xstrata vende mina de cobre Las Bambas en US\$ 5,850 millones. $13 / 4 / 2014$.

Gestión (2016). El avance del cobre en Perú y el estancamiento de Chile. 19/8/2016.

Glencore (2012). Antapaccay: del proyecto a la operación. Lima: Glencore.

Global Witness (2014). Peru’s Deadly Environment. Londres: Global Witness.

Gobel, B. y Ulloa, A. (eds.) (2016). Extractivismo minero en Colombia y América Latina. Berlín y Bogotá: Ibero-Amerkanisches Institut y Universidad Nacional de Colombia. Recuperado de http://www.desigualdades.net/Resources/Publications/Extractivismominero-Goebel_Ulloa.pdf

Gudynas, E. (2016a). Natural resource nationalisms and the Compensatory State in progressive South America. En P.A. Haslam y P. Heidrich (eds.), The Political Economy of Natural Resources and Development: From Neoliberalism to Resource Nationalism (pp. 103-117). Londres y Nueva York: Routledge.

Gudynas, E. (2016b). Teología de los extractivismos. Tabula Rasa, 24, 11-23. https://doi.org/ $10.25058 / 20112742.55$

Gudynas, E. (2017). Los ambientalismos frente a los extractivismos. Nueva Sociedad, 268.

Haarstad, H. (ed.) (2012). New political spaces in Latin American natural resource governance. Nueva York: Palgrave Macmillan. https:/doi.org/10.1057/9781137073723

Humpel, E. (2011). Monitoreo ambiental participativo en la provincia de Espinar. Elaboración de una línea de base en el ámbito del proyecto Xstrata-Tintaya. Cusco: Vicaría de la Solidaridad de Sicuani, Misereor.

INS, Censopas, Diresa Cusco (2010). Línea de base en salud de las comunidades afectadas por el proyecto minero quechua, Cusco - Espinar 2010. Lima: INS.

Instituto de Defensa Legal (2012). Informe: la criminalización de las protestas sociales durante el primer año del gobierno de Ollanta Humala, de la gran transformación a la mano dura. Lima: IDL-Justicia Viva.

Instituto de Defensa Legal (2015). Afirman que comunera de Espinar sí falleció por contaminación con cadmio y arsénico. 20/8/2015.

Kothari, U. y Cooke, B. (2001). Power, knowledge and social control in participatory development. En B. Cooke y U. Kothari, Participation: The new tyranny? (pp. 139-152). Londres: Zed Books.

La Mula (2013). Espinar: MINAM reconoce problemas ambientales y sanitarios. 11/4/2013.

La República (2012). Protestas en Espinar degeneraron en muertes. 29/5/2012. 
La República (2015a). Mesa de Diálogo generó descontento en Espinar y denuncia contaminación. 11/2/2015.

La República (2015b). Gobierno ocultó evidencia de contaminación en niños y adultos de Espinar. 24/11/2015.

Ley 28611. Ley General del Ambiente. Ministerio del Ambiente.

Maldonado, E. (2013). Sistema Nacional de Prevención y Gestión de Conflictos Sociales. Revista Quehacer. Lima: Desco.

Noticias Ser (2013). Desconfianza y preocupación en Espinar frente al monitoreo ambiental liderado por el MINAM. 17/4/2013.

Núñez, V., Ampie, M. Ortegón, J., Rodriguez, M., Dias, R., Oliva, B., ... Santos, J. (2015). Criminalización de defensores de derechos humanos en el contexto de proyectos industriales: un fenómeno regional en América Latina. Ciudad: FIDH. Recuperado de https://www.fidh.org/IMG/pdf/criminalisationobsangocto2015bassdef.pdf

Oficina Nacional de Diálogo y Sostenibilidad (2013). Institucionalizando el diálogo a un año de gestión. Experiencias y aportes de la ONDS-PCM 2012-2013. Lima: PCM.

Oficina Nacional de Diálogo y Sostenibilidad (2016). Memoria I Encuentro Internacional sobre Diálogo, Industrias Extractivas y Conflictividad Social: Derechos Humanos y Empresa. Lima: PCM.

Panfichi, A. (ed.) (2007). Participación ciudadana en el Perú: disputas, confluencias y tensiones. Lima: PUCP.

Paredes, M. (2015). Representación política indígena: un análisis comparativo subnacional. Lima: IEP.

Paredes, M. y De La Puente, L. (2014). Protestas y negociaciones socioambientales. El caso de las industrias extractivas. En G. Damonte y G. Vila, Agenda de investigación en temas socioambientales en el Perú: una aproximación desde las ciencias sociales (pp. 75-105). Lima: PUCP.

Paredes, M. y L. De la Puente (2017). The Social Construction of State Problems, the role of the Ombudsman in building institutions for extractive conflict. En E. Dargent (et al.), Resource booms and institutional pathways: the case of extractive industries Peru (pp. 119-151). Cham, Suiza: Palgrave MacMillan. https://doi.org/10.1007/ 978-3-319-53532-6_5

Perreault, T. (2013). Dispossession by accumulation? Mining, water and the nature of enclosure on the Bolivian Altiplano. Antipode, 45(5), 1050-1069. https://doi.org/ 10.1111/anti.12005

Perreault, T. y Martin, P. (2005). Geographies of neoliberalism in Latin America. Environment and Planning, 37, 191-201. https://doi.org/10.1068/a37394

Peters, G. (2012). Governance as Political Theory. En D. Levi-Faur (ed.), The Oxford Handbook of Governance (¿pp-pp ?). Oxford: Oxford University Press. https:/doi. org/10.1093/oxfordhb/9780199560530.013.0002

RM 164-2012-PCM. Conformación del Grupo de Trabajo «Mesa de Diálogo para solucionar la problemática socioambiental existente en la provincia de Espinar». Presidencia del Consejo de Ministros. 
Rojas, P. y A. Leiva (2012). Sistematización de los monitoreos y evaluaciones realizadas en la provincia de Espinar sobre presencia de metales pesados en el ambiente y en la salud de las personas. Lima: MUQUI, CooperAcción, FEDEPAZ.

Sanborn, C., Hurtado, V. y Ramírez, T. (2016). La consulta previa en el Perú: avances y retos. Lima: Universidad del Pacífico. https://doi.org/10.21678/978-9972-57-366-8-2016

Scott, J.C. (1998). Seeing like a state: How certain schemes to improve the human condition have failed. New Haven, CT: Yale University Press.

Secretaría Técnica de la Mesa de Diálogo de Espinar (2013). Informe final de la mesa de diálogo de Espinar.

Servindi (2016). Contaminación en Espinar llegó a la CIDH. 11/4/2016.

Svampa, M. (2013). Consenso de los commodities y lenguajes de valoración en América Latina. Nueva sociedad, democracia y política en América Latina.

Svampa, Mariestella (2008). La disputa por el desarrollo: territorio, movimientos de carácter socioambiental y discursos dominantes. Recuperado de http://www.maristellasvampa.net/archivos/ensayo43.pdf

Temper, L., Walter, M., Rodriguez, I., Kothari, A. y Turham, E. (2018). A perspective on radical transformations to sustainability: resistances, movements and alternatives. Sustainability Science, 13(3), 747-764. https://doi.org/10.1007/s11625-018-0543-8

Thorp, R. (2012). The challenges of mining-based development in Peru. En R. Thorp, S. Battistelli, Y. Guichaoua, J.C. Orihuela y M. Paredes, The developmental challenges of mining and oil: lessons from Africa and Latin America (pp. 110-130). Nueva York: Palgrave Macmillan.

Valencia, A. (2017). Participación ciudadana en la gestión ambiental del sector minero en el Perú: reflexiones para la incorporación de una mirada de género. Cuadernos de Investigación (3). Lima: Escuela de Gobierno y Políticas Públicas de la Pontificia Universidad Católica del Perú.

Vila, G. (2014). Naturaleza intervenida: una mirada a las construcciones de la naturaleza desde la ecología política. En G. Vila y G. Damonte (eds.), Agenda de investigación en temas socioambientales en el Perú: una aproximación desde las ciencias sociales (pp.193-221). Lima: PUCP.

Willaqniki (2013). Mesas de diálogo, mesas de desarrollo y conflictos sociales en el Perú. Willaqniki. Informe de diferencias, controversias y conflictos sociales, 5. Lima, PCM-ONDS.

Willaqniki (2014). Casos emblemáticos: Morococha y Espinar. Willaqniki. Informe de diferencias, controversias y conflictos sociales, 15. Lima, PCM-ONDS.

Zeisser, M. (2015) La experiencia de la mesa de diálogo en Espinar (2012-2013). Lima: Ford Foundation, Oxfam, CooperAcción. 